\title{
Fast in-situ tool inspection based on inverse fringe projection and compact sensor heads
}

\author{
Steffen Matthias ${ }^{\mathrm{a}}$, Markus Kästner ${ }^{\mathrm{a}}$, and Eduard Reithmeier ${ }^{\mathrm{a}}$ \\ ${ }^{a}$ Institute of Measurement and Automatic Control, Leibniz Universität Hannover, Nienburger \\ Str. 17, 30167 Hannover, Germany
}

\begin{abstract}
Inspection of machine elements is an important task in production processes in order to ensure the quality of produced parts and to gather feedback for the continuous improvement process. A new measuring system is presented, which is capable of performing the inspection of critical tool geometries, such as gearing elements, inside the forming machine. To meet the constraints on sensor head size and inspection time imposed by the limited space inside the machine and the cycle time of the process, the measuring device employs a combination of endoscopy techniques with the fringe projection principle. Compact gradient index lenses enable a compact design of the sensor head, which is connected to a CMOS camera and a flexible micro-mirror based projector via flexible fiber bundles. Using common fringe projection patterns, the system achieves measuring times of less than five seconds. To further reduce the time required for inspection, the generation of inverse fringe projection patterns has been implemented for the system. Inverse fringe projection speeds up the inspection process by employing object-adapted patterns, which enable the detection of geometry deviations in a single image. Two different approaches to generate object adapted patterns are presented. The first approach uses a reference measurement of a manufactured tool master to generate the inverse pattern. The second approach is based on a virtual master geometry in the form of a CAD file and a ray-tracing model of the measuring system. Virtual modeling of the measuring device and inspection setup allows for geometric tolerancing for free-form surfaces by the tool designer in the CAD-file. A new approach is presented, which uses virtual tolerance specifications and additional simulation steps to enable fast checking of metric tolerances. Following the description of the pattern generation process, the image processing steps required for inspection are demonstrated on captures of gearing geometries.
\end{abstract}

Keywords: Fringe Projection, Ray-Tracing, Inspection, Endoscopy, Single-shot, Tolerance checking

\section{INTRODUCTION}

In order to avoid the production of defective parts, tool inspection is a critical part of modern production processes. As an example, the sheet-bulk metal forming process aims to produce parts with deep drawing and bulk forming elements in a single process step. Both due to the complexity of the tool geometry and the dimensions of the forming press assembly, space for measuring systems is limited. Additionally, with cycle times of in the order of one second, time spend on tool inspections must be kept low. To meet both requirements, a new measuring system is being researched, which aims to perform inline inspection of critical tool geometries, such as gearing elements, in a short time. The new fiber-optic fringe projection system employs a combination of endoscopy techniques with the fringe projection principle ${ }^{1}$ to achieve both compact dimensions of the sensor head as well as short measuring times. Fig. 1 shows a close up photo of the sensor head and the forming tool.

By using conventional fringe projection pattern sequences, the system is capable of measuring 3-D point clouds of tool geometries in less than $3 \mathrm{~s}$. To improve the capabilities of the system for inline-inspection of industrial processes, also the inverse fringe projection principle has been adapted, which allows for the detection of deviations from a reference geometry by projection a single pattern, which ideally features parallel stripes in the camera image. This special pattern is obtained in an initial calibration step, for example by measuring a

Further author information:

E-mail: steffen.matthias@imr.uni-hannover.de, Telephone: +49 5117624456

Optical Metrology and Inspection for Industrial Applications IV, edited by Sen Han, Toru Yoshizawa, Song Zhang, Proc. of SPIE Vol. 10023, 100230K · C 2016 SPIE · CCC code: 0277-786X/16/\$18 · doi: 10.1117/12.2247482 


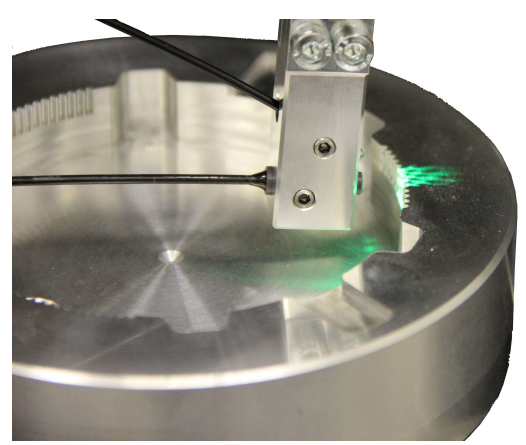

Figure 1. Sensor head next to forming tool

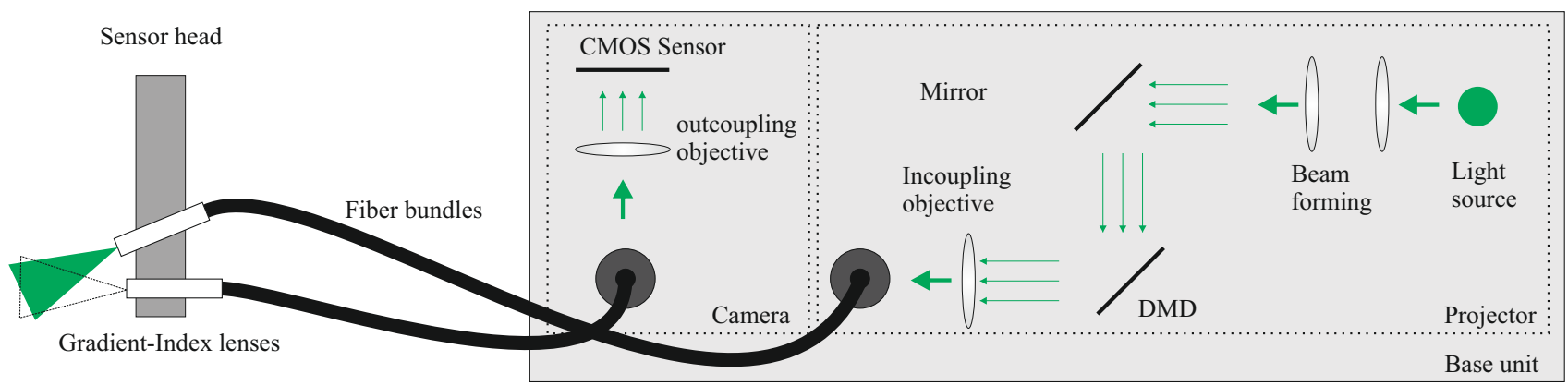

Figure 2. Schematic of the fiber-optic fringe projection system

manufactured master geometry ${ }^{2} .{ }^{3}$ By using a virtual reference, for example 3-D geometry from a CAD-file, the inverse patterns may be obtained by simulation. ${ }^{4}$ While the described methods allow for the fast detection of deviation from the reference geometry, checking for tolerances is problematic. In, ${ }^{5}$ quantification of the deviations are calculated by an additional processing step based on a linearization of the phase-3-D-relation around the reference geometry. In this work, a new approach is presented, which uses a virtual representation of the metric tolerances in a CAD file to generate deviation thresholds by an additional simulation step, allowing for very fast evaluation of inverse projected patterns, while not relying on linearization to check the tolerances.

Based on a short introduction of the fiber-optic fringe projection system, first the methods used for the inverse simulation are presented, followed by a short description of the generation of inverse patterns based on a manufactured master. After an explanation of the tolerance specification and threshold calculation used for the fast tolerance checking for free-form surfaces, the evaluation of inversely projected patterns is explained. Finally, results are shown for the inspection of gearing geometries on a forming tool of a sheet-bulk metal forming process.

\section{FIBER-OPTIC FRINGE PROJECTION}

The fiber-optic fringe projection system consists of a base-unit, which houses the pattern generator and camera unit, and a compact sensor head. Both elements are coupled using two flexible high-resolution image fibers (100.000 pixels) with a diameter of $1.7 \mathrm{~mm}$ supplied by Fujikura Ltd. (type FIGH-100-1500N). A schematic of the system is shown in Fig. 2. The sensor head features gradient-index (GRIN) optics by GRINtech with diameter of $2 \mathrm{~mm}$ and a working distance of $10 \mathrm{~mm}$, resulting in a measuring volume of about $5 \mathrm{~mm} \times 5 \mathrm{~mm} \mathrm{x}$ $2.5 \mathrm{~mm}$. For larger measuring volumes GRIN optics with larger working distances are applicable. The camera part of the base unit consists of a 4x microscope objective for fiber coupling and a Point Grey GS3-U3-23S6M-C camera with a resolution of 1920 x 1200 pixels. For the projector part, two different light sources are available. A LED is employed as an incoherent light source with higher beam divergence in comparison with a laser light source, while the latter suffers from speckle artifacts. ${ }^{6}$ The flexible generation of patterns is especially of use for the projection of object adapted inverse fringe projection patterns. Depending on the optical properties of the specimen, full 3-D measurements of geometries can be obtained in less than $3 \mathrm{~s}$, while standard deviation of point-reference distances for a calibrated cylinder geometry with a radius of $1 \mathrm{~mm}$ is around $6 \mu \mathrm{m} .^{7}$ 


\section{SYSTEM MODEL}

In order to simulate inverse patterns for faster tolerance checking, an accurate virtual model of the fringe projection system and the scene is required. The system model describes optical parameters of the camera and projector, such as focal length and the position of the optical axis on the pixel matrix, as well as geometric parameters, for example distance of camera to projector and the triangulation angle. The scene consists of the virtual specimen and its position and orientation relative to the fringe projection system. In this section, the pinhole model and distortion models of camera and projector will be explained. The parametric model of a fringe projection system, sometimes also called active stereo, is identical to a stereo camera.

Equation 1 shows the matrix formulation of perspective projection, where $f_{1}$ and $f_{2}$ are the focal length of the model, $\alpha$ the skew factor, modeling non-orthogonal pixels, and $c_{x}$ and $c_{y}$ the position of the optical axis in the sensor coordinate system. It needs to be noted, that $f_{1}$ and $f_{2}$ include both the focal length of the optics and the size of the pixel in each dimension.

$$
K=\left[\begin{array}{ccc}
f_{1} & \alpha & c_{x} \\
0 & f_{2} & c_{y} \\
0 & 0 & 1
\end{array}\right]
$$

The sensor coordinate $(u, v)$ of a given object $X$ point in the camera reference can be obtained frame by choosing $s$ equally to the point's z-coordinate, according to equation 2.

$$
s\left[\begin{array}{l}
u \\
v \\
1
\end{array}\right]=K X
$$

If an object point is given in an arbitrary world reference frame, it must be transformed to the camera reference frame by applying a rigid transformation. The transformation followed by a projection to the sensor plane is given by equation 3 , where $R$ is a $3 \times 3$ rotation matrix and $t$ a $3 x 1$ translation vector.

$$
s\left[\begin{array}{l}
u \\
v \\
1
\end{array}\right]=K(R X+t)
$$

Lens distortions of the optical system are modeled in image space by a distortion function (equation 4).

$$
\left[\begin{array}{l}
u_{\text {dist }} \\
v_{\text {dist }}
\end{array}\right]=f(u, v)
$$

Radial distortion artifacts, such as barrel or pincushion distortion, can be approximated by polynomials, while distortions from misaligment of optical elements are described using a decentering term. The following equations describe the Brown distortion model: ${ }^{8}$

$$
\begin{gathered}
r^{2}=\left(u-c_{x}\right)^{2}+\left(v-c_{y}\right)^{2} \\
u_{\text {dist }}=u\left(1+k_{1} r^{2}+k_{2} r^{2}+k_{3} r^{6}\right)+\left(l_{1}\left(r^{2}+2 u^{2}\right)+2 l_{2} u v\right) \\
v_{\text {dist }}=v\left(1+k_{1} r^{2}+k_{2} r^{2}+k_{3} r^{6}\right)+\left(l_{2}\left(r^{2}+2 v^{2}\right)+2 l_{1} u v\right)
\end{gathered}
$$

Parameters $k_{1}, k_{2}$ and $k_{3}$ are the coefficients of the radial distortion, $l_{1}$ and $l_{2}$ the coefficients for decentering. Coordinates $(u, v)$ describe the indices on the virtual undistorted image, $\left(u_{\text {dist }}, v_{\text {dist }}\right)$ the distorted coordinates on the camera sensor.

Both camera and projector of the fiber-optic fringe projection system are modeled using the pinhole model and the Brown distortion model when using the unmodified GRIN lenses. It needs to be noted, that the parameters of camera and projector differ, as pixel sizes and the optical systems are not identical. Therefore, the index $c$ is used for camera parameters and index $p$ for projector parameters in the next section. For the fiber-optic system, parameters of the model are obtained by automated positioning of a point pattern standard by a linear stage in the measuring volume. ${ }^{9}$ 


\section{INVERSE SIMULATION}

As described in the previous section, the simulation of inverse pattern is based on a virtual representation of the fringe projection system as well as the scene, consisting of the virtual object and its relative pose and position to the fringe projection system. A ray-tracing ${ }^{10}$ algorithm is used to simulate the illumination of the specimen by the projector as well as the capturing process of the camera. A key part of the ray-tracing process is using equation 2. If multiplied with the inverse projection matrix $K^{-1}$ on both sides, a 3-D line equation is obtained for each pixel:

$$
X=s K^{-1}\left[\begin{array}{l}
u \\
v \\
1
\end{array}\right]
$$

By intersecting the line with arbitrary virtual objects in the camera coordinate system, virtual camera images can be rendered. As the model of camera and projector are identical, the same is valid for the projector.

For compatibility with CAD software, the virtual simulation developed for the fiber-optic fringe projection system expects the virtual object to be represented as triangle data in an STL-format file. While exporting the STL file from CAD-software, the parameters of the surface triangulation have to be chosen appropriately to avoid significant deviations to the parametric surfaces. As the coordinate system used in the CAD file is different to the coordinate system of the measuring device, a referencing step is required. This is performed by measuring geometric markers on the real geometry in the desired pose and calculating the coordinate transform $T_{\text {object }}$ from the CAD-coordinate system to the camera coordinate system. Additionally, the system parameters described in section 3 need to be available. The parameters obtained by calibration are the projection matrix of the camera $K_{c}$, the projection matrix of the projector $K_{c}$ and the rigid transformation from camera to projector described by matrix $T_{c p}$. As the GRIN lenses used in projector and camera feature significant distortion artifacts, approximations must be calibrated by the functions $f_{d, c}\left(u_{c}, v_{c}\right)$ for the camera and $f_{d, p}\left(u_{p}, v_{p}\right)$ for the projector.

During the simulation, also the inverse distortion $f_{d, p}^{-1}$ for the projector is required, which is approximated by using the Gauss-Newton method to find the undistorted pixel coordinates $\left(u_{p}, v_{p}\right)$ which fulfill equations 6 and 7. The distorted pixel coordinates $\left(u_{p, d i s t}, u_{p, d i s t}\right)$ are used as the starting point for the optimization.

In order to calculate inverse patterns with the desired shape in the camera images, projector-camera correspondences need to be calculated. This is achieved by performing the following simulation steps:

1. Transform virtual geometry vertex data to the projector coordinate system using $T_{o b j e c t}$ and $T_{c p}$.

2. Calculate the corresponding undistorted coordinates for the full projector matrix with $f_{d, p}^{-1}$.

3. Cast rays from the undistorted virtual projector pixel matrix using the inverse projection matrix $K_{p}^{-1}$.

4. Calculate intersection points with the virtual geometry in projector coordinates with ray-triangle intersection. For multiple intersections on individual rays, store only the intersections closest to the origin of the projector coordinate system.

5. Transform intersection points to camera coordinate system using the inverse transformation $T_{c p}^{-1}$.

6. Transform virtual geometry vertex data to camera coordinate system using $T_{\text {object }}$.

7. Build rays from camera origin to hits and intersect with virtual geometry to detect occlusions and remove invisible points.

8. Calculate reflected intensity according to illumination model based on surface normals and the direction of projector and camera rays (optional).

9. Project points onto virtual camera pixel matrix using $K_{c}$.

10. Calculate distorted camera pixel coordinates using $f_{d, c}$. 
The outcome of the simulation is a lookup-table with projector-camera correspondences in the form of a lookup table. For each projected pixel $\left(u_{p, d i s t}, v_{p, d i s t}\right)$ either a corresponding point $\left(u_{c, d i s t}, v_{c, d i s t}\right)$ on the camera matrix is defined or it is masked as invalid, which means that it either did not project onto the virtual specimen or it is occluded by the specimen's geometry in the camera image. After defining the desired camera image, usually a fringe pattern with parallel stripes, the projector pattern can be sampled from the camera image used the lookup table obtained by simulation. Pixels on the projector matrix that are masked as invalid will be set to zero intensity.

A number of features of the simulation need to be considered:

- Lens distortion of both camera and projector are fully compensated by pre-distorting the projector pattern. Given an ideal calibration of the system and an ideal specimen, the patterns will appear exactly as chosen.

- The simulation is calculated using floating point arithmetic, thus the simulated camera points not be integer values. The camera matrix can either be sampled using suitable interpolation (such as bilinear interpolation), or directly sample from the function used to generate the desired camera image for higher precision. As usually cosine-patterns are used, the last option should be preferred.

- The ray-tracing process is started from the virtual projector, the complete projector matrix is sampled discretely to avoid the need for interpolation in projector pixel coordinates.

- Reflection properties of the surface can be qualitatively described for example by the Phong illumination model, ${ }^{11}$ which defines the diffuse and specular fractions of surface reflectivity. More advanced models exist, at the cost of increased computation times. Due to highly variable influences of the forming process, such as scratches or lubricants, an accurate modeling of the per-pixel reflection of the tool surface is impossible. However, already the simple Phong model can give an estimation of highlights, helping to avoid direct reflection on surfaces with highly specular reflection. While effects like inter-object reflections are possible to implement using multiple rays per pixel and reflection, ${ }^{12}$ these techniques are not used in the following experiments.

\section{DIRECT PATTERN INVERSION}

In contrast to the virtual calculation of projector-camera correspondences for generating the inverse patterns, the correspondences can be obtained directly by phase measurements, if a manufactured master tool geometry is available. If absolute phase maps are measured for fringe patterns in both vertical and horizontal direction of the projector, the correspondences can be found by matching the absolute phase on the camera matrix with the absolute phase on the projector matrix without modeling and calibration of the measuring device. While this approach simplifies the generation of inverse patterns, it has a number of significant drawbacks:

- Very precise manufacturing of the master geometry is required.

- For the generation of the inverse pattern, the master geometry needs to be positioned exactly like the geometry to be inspected.

- Per-pixel noise in the phase-measuring process is present as interference in the inverse reference pattern.

- No virtual tolerancing is possible, in order to obtain metric deviations modeling and calibration of the system is required, similar to virtual simulation.

- Additional processing steps are required to check for tolerances, metric deviations are usually only checked by linear approximation.

Proc. of SPIE Vol. 10023 100230K-5 


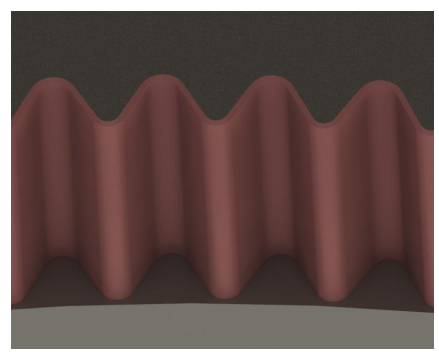

Figure 3. Render of exemplary virtual tolerance band

\section{GEOMETRIC TOLERANCING}

In order to enable tolerance checking via virtual inverse fringe projection, tolerances need to be defined on the virtual geometry. For the new approach presented in this article, the tolerances are defined by two additional virtual geometries. The first geometry describes the lower tolerance limit defined in the CAD file, while the second defines the upper tolerance limit. Effectively, the lower tolerance limit virtually represents the tool in the state of maximum tolerable abrasion, and the upper tolerance limit the tool in the state of maximum tolerable material pick-up. An alternative interpretation of the two additional virtual surfaces is the definition of a tolerance band or envelope for the free-form surface in 3D. Fig. 3 shows an exemplary tolerance band drawn next to gearing geometries on a sheet-bulk metal forming tool.

The two additional virtual geometries are used to simulate the deformation of the reference inverse pattern when projected on the upper and lower tolerance limit, resulting in two virtual camera images representing the maximum deformation of the reference pattern for the tolerance limits. After an evaluation of the projected pattern on a real specimen, the simulated camera patterns define a per-pixel threshold to perform tolerance checking in the captured images.

The simulation of the virtual camera images is similar to the process explained in section 4. Two simulation runs are needed, one with the lower tolerance geometry and one with the upper tolerance geometry. In order to generate the virtual camera images, the simulated lookup tables are used to sample the inverse projector pattern.

\section{PATTERN EVALUATION AND TOLERANCE CHECKING}

Morphological evaluations cite To detect deviations of the projected inverse pattern, robust evaluation by signal processing is required. Usually patterns with a cosine intensity profile are used as the deformation of the fringes can be obtained by phase analysis. These patterns are also employed for performing inspection with the fiberoptic fringe projection system.

When projecting the inverse pattern of the projector, the normalized intensity in the camera image can be described according to equation 9 . The fringe pattern features the frequency $\omega_{\text {fringe }}$ and is in this example oriented in vertical direction, with local phase modulation of $\Delta \phi$ if the specimen deviates from the reference. Parameter $A$ describes the surface reflectance in camera coordinates and is for an ideally diffuse object constant.

$$
I(u, v)=A(u, v) \cdot\left(0.5+0.5 \cdot \cos \left(\omega_{\text {fringe }} \cdot v+\Delta \phi(u, v)\right)\right)
$$

In order to extract the local phase deviations from the images, three different approaches may be considered. Patterns with a known global phase-shift, similar to conventional fringe projection, may be used for a robust evaluation of the local phase. For this, at least three patterns need to be projected. The process is robust towards interfering light from external sources and variations in surface reflectance. Equation 10 shows the phase evaluation algorithm, where $N$ is the total number of equally phase-shifted patterns projected and $n$ the index of an individual pattern image. By substracting the reference camera pattern phase and wrapping to a range of $-\pi \cdots \pi$, the local deviations can be recovered .

$$
\phi(u, v)=\arg \left(\sum_{n=1}^{N} I_{n}(u, v) \cos \left(n \frac{2 \pi}{N}\right)+i \cdot \sum_{n=1}^{N} I_{n}(u, v) \sin \left(n \frac{2 \pi}{N}\right)\right)
$$


When using a single pattern, evaluation of the local phase is enabled by applying the Hilbert transform line-wise to the measured intensity profile in the direction of the fringe pattern:

$$
\phi(u, v)=\arg (I(u, v)+i \cdot \mathcal{H}\{I(u, v)\})
$$

However, when using the single pattern method, a number of constraints need to be met:

- The signal $I$ must not feature a constant component.

- Interference from external light sources and surface reflectance must be appropriately suppressed.

In the case of a mostly diffuse reflective specimen, the background in the camera image can be suppressed effectively by high-pass filtering. The local phase is obtained by applying the Hilbert transform on the image in direction of the fringe pattern.

Considering equation 9 , both constraints can be met for mostly diffuse reflecting specimen if a high-pass filter is applied, as $A$ will be approximately constant over the complete image. With varying reflectance of the specimen, the spectrum of $A$ spreads to higher frequencies. If the spectrum of $A$ is overlapping with the modulated pattern, the phase according to equation 11 can only be recovered with errors as a result from interference. Due to the overlap in the spectrum, high-pass filtering cannot remove this interference.

Thus, if overlap of the spectrum of the surface reflectance with the spectrum of the modulated pattern cannot be avoided, the single pattern method will lead to inaccurate results when performing tolerance checking. The introduction of a second pattern, which features the inverted intensity of the reference pattern, helps to suppress the influence of the background:

$$
I_{i n v}(u, v)=A(u, v) \cdot\left(0.5-0.5 \cdot \cos \left(\omega_{\text {fringe }} \cdot v+\Delta \phi(u, v)\right)\right)
$$

By substracting the camera capture of the projected inverse pattern from the camera capture of the reference pattern, the influence of $A$ can be completely removed. Robust evaluation of the local phase can be performed by applying the Hilbert transform, as both constraints are met.

Several advantages from simulation of tolerance: The simulation of the deformation of the reference patterns on Also, the effect of surface reflectivity may be reduced by accurate modeling.

\section{RESULTS}

Fig. 4 shows the process of generating inverse patterns and tolerance checking outlined in the previous sections. In image a) a fringe pattern of a fringe projection sequence using parallel projector patterns is shown. The classical 3-D measurement is used to calculate the pose of the virtual reference object for inverse simulation. As a result from simulation, the inverse projector pattern is shown in subfigure b). By employing the two-pattern method outlined in the previous section, the phase map shown in subfigure d) is calculated. By comparing the measured phase deviations from the reference pattern and comparing to the tolerance thresholds obtained by the two additional simulation steps described in section 6, for each surface point can be checked if it is lying within the virtual tolerance band. For the inspection of the gearing geometries on the forming tool shown in Fig 4 , a virtual tolerance of $\pm 30 \mu \mathrm{m}$ in direction of the surface normals has been defined. Due to the nonlinearity of the fringe deformation, no metric quantification of the deviation within the tolerance band can be given. Thus, the deviations are shown linearized in percent in subfigure e). For visualisation, detected areas that are out-of-tolerance can be reprojected on the virtual reference, as shown in subfigure f).

\section{DISCUSSION}

The method described in the previous sections allows for very fast tolerance checking, as only a few number of patterns need to be projected by the measuring device. Additionally, processing times for the tolerance check are very low, as only two per-pixel thresholds need to be checked. All data required for performing inspection is automatically obtained by simulation, which as input only requires a 3 -D model of the specimen to 

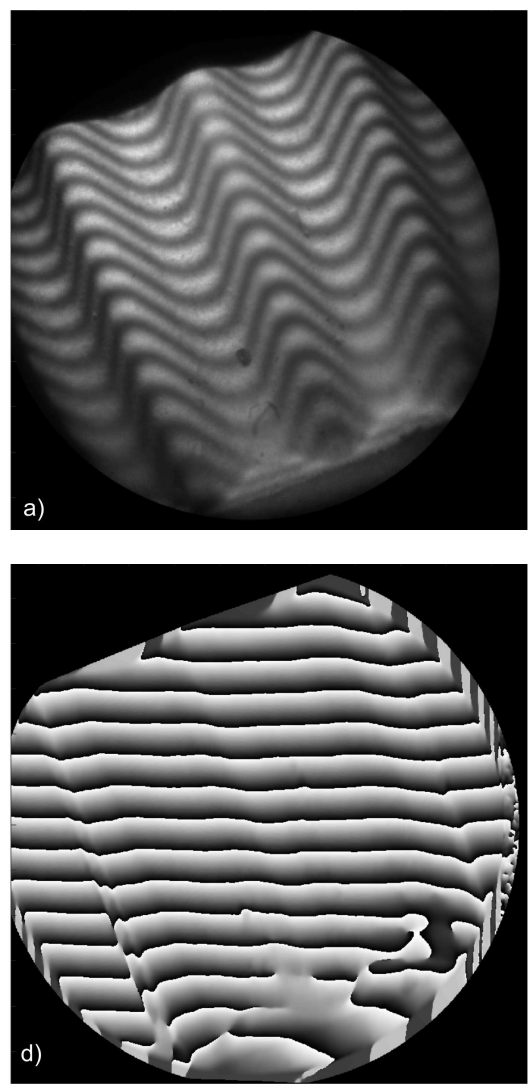
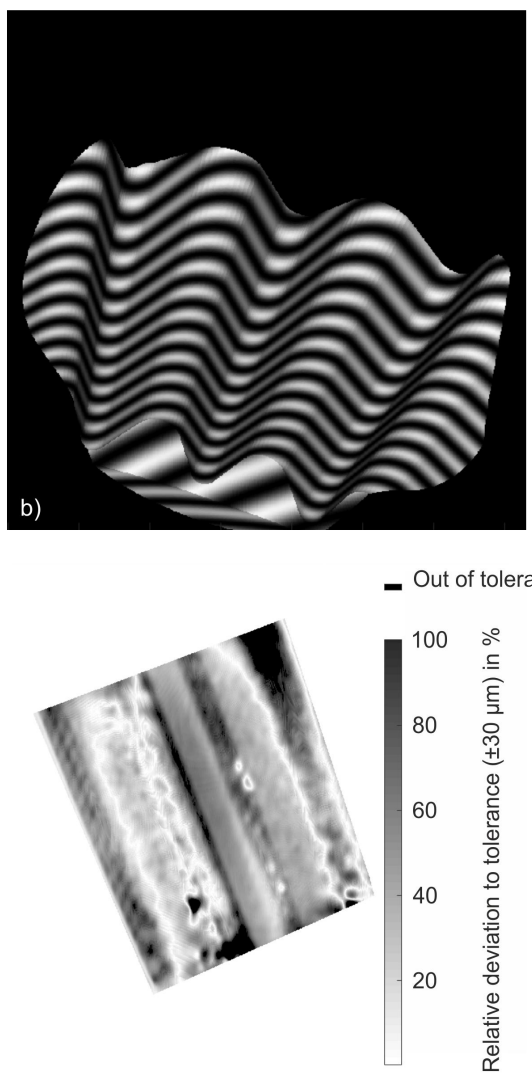
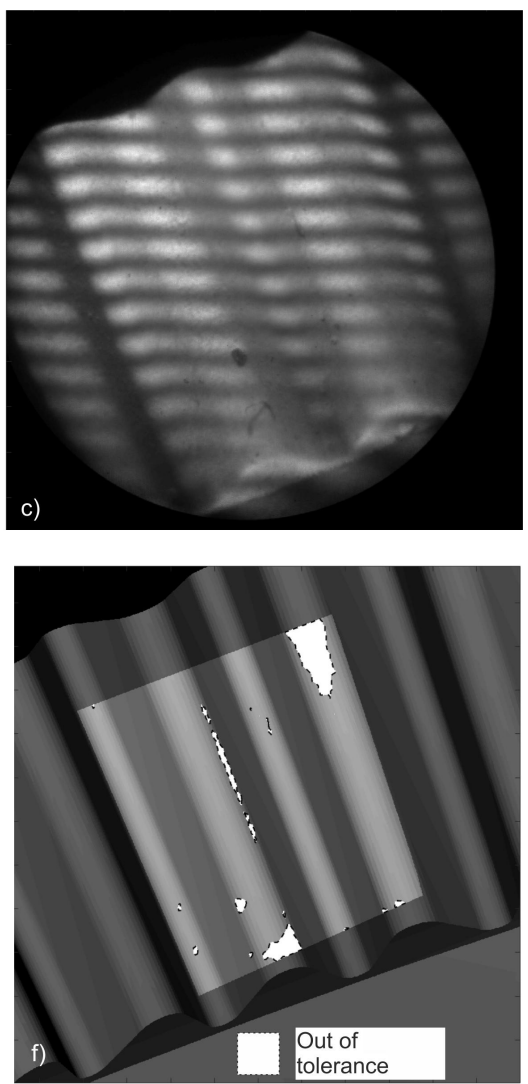

Figure 4. Process of generating inverse pattern and performing deviation analysis via inverse fringe projection. a) Conventional pattern projected onto specimen. b) Simulated inverse pattern for specimen. c) Inverse pattern projected onto specimen. d) Recovered phase image from 2 inverse projected patterns. e) Tolerance checking. f) Out-of-tolerance areas projected onto rendered specimen.

be inspected and a metric definition of the tolerances for each surface point, next to system calibration data and measuring pose. Compared to previous approaches, which performed quantified deviation analysis using linear approximation, the new approach features reduces processing times and more accurate tolerance checking. The check against the tolerances does not rely on linear approximation. Additionally, for surface points within the tolerance, the deviation from the reference geometry can be approximated linearly. However, tolerance checking for features such as radii or angles is not directly possible using this method. If the tolerance specifications for a feature can be converted to an envelope, the method can be applied.

In combination with the compact sensor head, the system is capable of performing inline inspection of the sheet-bulk metal forming process. Inspection times of less than one second are achieved using the two pattern method. Further reduction of the inspection process may be achieved by using single pattern processing, as described in section 7 , which will however lead to an increased uncertainty due to the varying reflectance of the technical surfaces used for the industrial process.

In general, inverse fringe projection allows for the fast detection of deviations from a reference geometry. However, precise positioning of the sensor head must be ensured, as the reference patterns obtained by simulation or direct inversion from measurements are only valid for a certain alignment of sensor and specimen. By simulating the camera image for geometries with deviations, both pattern frequency and filter design can be adapted to avoid phase unwrapping issues and reduce noise. 


\section{SUMMARY}

A new fringe projection system for the inspection of industrial processes has been presented. The system is capable of measuring For fast tolerance checking, a new approach has been demonstrated, which is based on a virtual representation of the measuring device and the specimen, including geometric tolerances. By introducing an additional simulation step, accurate and fast inline checking of tolerances for free-form surfaces is achieved without the need of linearization. After the generation of the inverse patterns, inspection can be performed in less than one second. While the projection of a single pattern is sufficient, it could be shown that the introduction of a second pattern greatly enhances the robustness of the method towards variances in reflectance, as present for technical surfaces.

\section{ACKNOWLEDGMENTS}

The authors would like to thank the German Research Foundation (DFG) for funding the project B6 "Endoscopic geometry inspection" within the Collaborative Research Center (CRC) / TR 73.

\section{REFERENCES}

[1] Gorthi, S. S. and Rastogi, P., "Fringe projection techniques: whither we are?," Optics and lasers in engineering 48(IMAC-REVIEW-2009-001), 133-140 (2010).

[2] Kalms, M. K., Osten, W., and Jueptner, W. P. O., "Inverse projected-fringe technique with automatic pattern adaption using a programmable spatial light modulator," in [International Conference on Applied Optical Metrology], 483-489 (1998).

[3] Li, W., Bothe, T., Osten, W., and Kalms, M., "Object adapted pattern projection-part i: generation of inverse patterns," Optics and lasers in engineering 41(1), 31-50 (2004).

[4] Pösch, A., Vynnyk, T., and Reithmeier, E., "Fast detection of geometry defects on free form surfaces using inverse fringe projection," Journal of the CMSC 8(1), 10-13 (2013).

[5] Bothe, T., Li, W., von Kopylow, C., and Juptner, W. P., "Object-adapted inverse pattern projection: generation, evaluation, and applications," in [Speckle Metrology 2003], 291-296 (2003).

[6] Matthias, S., Kästner, M., and Reithmeier, E., "Comparison of laser and led illumination for fiber-optic fringe projection," in [SPIE Photonics Europe], Berghmans, F. and Mignani, A. G., eds., SPIE Proceedings, 989905, SPIE (2016).

[7] Matthias, S., Loderer, A., Koch, S., Gröne, M., Kästner, M., Hübner, S., Krimm, R., Reithmeier, E., Hausotte, T., and Behrens, B.-A., "Metrological solutions for an adapted inspection of parts and tools of a sheet-bulk metal forming process," Production Engineering 10(1), 51-61 (2016).

[8] Brown, D. C., "Decentering distortion of lenses," Photometric Engineering 32(3), 444-462 (1966).

[9] Matthias, S., Kästner, M., and Reithmeier, E., "Evaluation of system models for an endoscopic fringe projection system," Measurement 73, 239-246 (2015).

[10] Glassner, A. S., [An introduction to ray tracing], Elsevier (1989).

[11] Phong, B. T., "Illumination for computer generated pictures," Communications of the ACM 18(6), 311-317 (1975).

[12] Shirley, P., Wang, C., and Zimmerman, K., "Monte carlo techniques for direct lighting calculations," $A C M$ Transactions on Graphics (TOG) 15(1), 1-36 (1996). 